\title{
The effect of STM parameters on tip-enhanced Raman spectra†
}

\author{
Natalia Martín Sabanés, (iD * Amala Elizabeth, Jonas H. K. Pfisterer \\ and Katrin F. Domke*
}

Received 8th May 2017, Accepted 12th May 2017

DOI: $10.1039 / c 7 f d 00164 a$

In this work, we evaluate the dependence of tip-enhanced Raman (TER) spectra of a monolayer of thiophenol at a Au(111) electrode on the scanning tunneling microscope's tunneling current set-point and bias voltage parameters. We find an increase of the TER intensity upon set-point increase or bias decrease as expected from a gap-distance reduction. The relations obtained follow a theoretical model considering a simple gap-distance change when tuning the mentioned parameters. We find that the value of the bias voltage affects the TER intensity to a larger extent than the current set-point. Therefore it is advisable to work in a low-bias regime when aiming for ultrasensitive TER measurements.

\section{Introduction}

In tip-enhanced Raman spectroscopy (TERS), the use of a scanning probe microscope tip as plasmonic antenna object yields the vibrational fingerprint of the system under study with sub-monolayer sensitivity and nanometric spatial resolution. ${ }^{\mathbf{1} 2}$ In the last decade, the capabilities of the technique in reaching single-molecule sensitivity with extremely high spatial resolution (in some cases in the sub-nm range) in air or in UHV conditions have been demonstrated. ${ }^{3-8}$ Recently, TERS in liquid and electrochemical conditions has been achieved by a few groups, overcoming the experimental challenges TER studies at solid/liquid interfaces entail, such as the expected enhancement decrease due to beam aberrations and focus point distortion at the solid/liquid interface. ${ }^{9-15}$ In-liquid experiments or/and single-molecule detection require particularly high Raman enhancement factors and are therefore usually performed with scanning tunneling microscopy (STM)-based TERS setups that typically provide enhancement factors that are a couple of orders of magnitude higher than atomic-force microscopy (AFM)-based TERS setups. ${ }^{16}$ 
One of the methods to maximize the enhancement in a TERS experiment is to minimize the gap distance between the tip and sample. ${ }^{16-20}$ The TERS intensity, $I_{\text {TERS }}$, is inversely proportional to the 8 th to 10 th power of the tip-sample separation: $:^{18,20}$

$$
I_{\mathrm{TERS}}^{\mathrm{norm}} \approx\left(1+\frac{d}{\rho}\right)^{-p}
$$

with gap distance $d$, dipole radius $\rho$ (approximately the tip radius) and $p=10$ for fully incoherent scattering.

In STM, the gap distance depends on the tunneling parameters, i.e. the tunneling current setpoint, $I_{\mathrm{t}}$, and the bias voltage between the tip and sample, $E_{\mathrm{b}}$. Electron transport between electrodes separated by a thin insulating film in the low bias range is described by Simmons' equation: ${ }^{21-24}$

$$
I_{\mathrm{t}}=\frac{E_{\mathrm{b}}}{R\left(z_{0}\right)} \times \mathrm{e}^{-\beta d} \Rightarrow d=\frac{1}{\beta} \ln \left(\frac{E_{\mathrm{b}}}{I_{\mathrm{t}} \times R\left(z_{0}\right)}\right)
$$

where $R\left(z_{0}\right)$ is the junction resistance at landing position $z_{0}$, and $\beta$ is the tunneling decay constant where $\beta=\frac{4 \pi}{h} \sqrt{2 m \phi}$, with work function or barrier height $\phi$, electron mass $m$ and Planck's constant $h$. According to eqn (2), the tip approaches the sample upon increasing $I_{\mathrm{t}}$ or decreasing $E_{\mathrm{b}}$. Combining eqn (1) and (2), we derive an expression relating the TERS intensity to the STM parameters in constant current mode experiments:

$$
I_{\mathrm{TERS}}^{\mathrm{norm}} \approx\left[1+\frac{1}{\rho \beta}\left[\ln \left(E_{\mathrm{b}}\right)-\ln \left(I_{\mathrm{t}}\right)-\ln \left(R\left(z_{0}\right)\right)\right]\right]^{-10}
$$

Expression (3) predicts the behaviour of the TER signal intensity as a function of the gap size as determined by the STM parameters. Despite its potential impact on increasing the TERS sensitivity, only a few studies have reported how TER spectra vary with the chosen $I_{\mathrm{t}}$ and $E_{\mathrm{b}}$ values (ref. 5, 13, 24 and 25) and a systematic study for both $I_{\mathrm{t}}$ and $E_{\mathrm{b}}$ under identical conditions (same tip, same sample) is still absent. In general, the expected trends were observed in individual studies, i.e. an increasing $I_{\mathrm{t}}$ results in a rise of the peak and background intensities as expected from the corresponding gap distance decrease, ${ }^{5}$ and a similar effect has been observed upon decreasing the bias voltage.$^{25}$ For a monolayer of azobenzene, an unexpected deviation from the STM-parameter dependent distance relation was observed where $I_{\text {TERS }}$ decreased with increasing bias voltages faster than would be expected from a mere gap size increase. ${ }^{24}$ The quantitative discrepancies between model and experiment were suggested to arise from molecular bending induced by the local electric field in the tunneling region created by $E_{\mathrm{b}}$. Hence, the relationship between $E_{\mathrm{b}}$ and $I_{\mathrm{TERS}}$ was suggested to be a convolution of different effects including the gap distance and the related variation of the near-field enhancement magnitude and molecular reordering.

In this paper, we systematically explore the influence of both $E_{\mathrm{b}}$ and $I_{\mathrm{t}}$ on the TERS response. We compare TERS studies in inert gas (Ar) and in water for a monolayer of thiophenol ( $\mathrm{PhS}$ ) adsorbed on a $\mathrm{Au}(111)$ single crystal. We analyse the trends of the intensity changes when varying $I_{\mathrm{t}}$ and/or $E_{\mathrm{b}}$. A theoretical model 
of these effects allows us to predict optimal STM parameters in TERS experiments to exploit maximum signal enhancement and thus experiment sensitivity.

\section{Materials and methods}

\subsection{TERS setup}

The home-built TERS setup consists of a commercial STM (Keysight Technologies $\mathrm{GmbH}$, formerly Agilent, 5420) coupled in a side-illumination configuration $\left(55^{\circ}\right.$ between the incoming beam and surface normal) with a red $\mathrm{He}-\mathrm{Ne}$ laser (632.8 nm; REO LSPR-3501, $35 \mathrm{~mW}$ maximum output power, linearly polarized). An Olympus $50 \times$ long-working-distance objective $(\mathrm{WD}=10.6 \mathrm{~mm}, \mathrm{NA}=0.5)$ focuses/collects the incident/scattered light. Optical elements (gray filters, mirrors, pinholes) were purchased from Thorlabs. Two filters (a Semrock RazorEdge ultra steep long-pass edge filter LP02-633RE-25 with a cut-off at $79 \mathrm{~cm}^{-1}$ and a Semrock RazorEdge Dichroic LPD02-633RU-25 with a cut-off at $156 \mathrm{~cm}^{-1}$ ) in the detection path filter out Rayleigh scattering. A Horiba iHR 550 spectrograph with a nitrogen-cooled CCD camera (Symphony II, Horiba) is used as a detector. The optical setup is mounted on an optical table with active vibration isolation. The incoming laser is focused to the tip apex by $x, y$, and $z$ piezo stages (custom-made by Steinmeyer Mechatronics, formerly Feinmess Dresden, $3 \mathrm{~nm}$ precision) that independently move the STM in the $x$ and $y$ directions and the objective in the $z$ direction. Liquid experiments are performed by mounting the sample in a homebuilt liquid cell. Electrochemically etched Au tips are coated with an insulating layer of Zapon and used for the Ar and liquid experiments. Further details about the optical setup, liquid cell and tip preparation are given elsewhere. ${ }^{13}$

\subsection{Sample preparation}

Ethanol (purity 99.9\%), thiophenol and $\mathrm{H}_{2} \mathrm{O}_{2}$ (18 304, 34.5-36.5\%) from SigmaAldrich, sulfuric acid (purity $\geq 95 \%$ ) from FisherChem and ultrapure water (Millipore-Q, Milli-Q, resistivity over $18 \mathrm{M} \Omega$ ) are used in the experiments. To avoid organic contamination during the experiments, we clean all the glassware, teflon tools, liquid cells and substrates by immersing them overnight in piranha solution $\left(\mathrm{H}_{2} \mathrm{SO}_{4} / \mathrm{H}_{2} \mathrm{O}_{2}, 3: 1\right)$ followed by extensive rinsing in boiling Milli-Q water. A $\mathrm{Au}(111)$ single crystal of $5 \mathrm{~N}$ purity (Mateck) is used as the substrate and prepared according to Clavilier's method. ${ }^{26}$ The annealed substrate is immersed into $1 \mathrm{mM}$ ethanolic PhS solution for 8 hours and rinsed extensively afterwards to remove adsorbate multilayers. The sample is mounted immediately after preparation. A gentle flow of $\operatorname{Ar}(5 \mathrm{~N}$, Westfalen) is poured over the sample during the experiment (except during spectral acquisition) to prevent contamination.

\subsection{Experimental procedure and analysis}

Ar and water experiments were performed on different days and with several different tips following the same procedure and preparation. For each experiment, different series of $I_{\mathrm{t}}\left(E_{\mathrm{b}}\right)$ ramps at constant $E_{\mathrm{b}}\left(I_{\mathrm{t}}\right)$ are taken. Tuning $E_{\mathrm{b}}$ in our STM is achieved by grounding the tip and ramping the sample potential. Due to technical constraints, tuning the bias voltage by changing the potential of the tip while keeping the sample potential constant is only possible in the electrochemical mode. Each experimental series consists of TER spectra taken at 
different values of $I_{\mathrm{t}}$ (or $E_{\mathrm{b}}$ ) cycled at least once from high to low values and vice versa. $I_{\mathrm{t}}$ is ramped between 0.1 and $1.8 \mathrm{nA}$ with a constant $E_{\mathrm{b}}$ of $0.02 \mathrm{~V}, 0.04 \mathrm{~V}$ or 0.1 V. $E_{\mathrm{b}}$ is ramped between 0.02 and $0.5 \mathrm{~V}$ at a constant $I_{\mathrm{t}}$ of $1.3,0.8$ or $0.3 \mathrm{nA}$. For each combination of values, 5 consecutive spectra are taken and the average used for fitting purposes. The STM parameters are ramped under tunneling conditions, and all the spectra in one series are taken at the same location on a flat terrace of the $\mathrm{Au}(111)$ substrate as determined by STM topographic imaging prior to the experiment, with the same far-field focussing conditions. The far-field spectra (acquired by retracting the tip $20 \mathrm{~nm}$ from the tunneling position) are taken several times during a series by disabling the feedback mechanism of the STM (no motor retraction) to prove the cleanliness and stability of the tip during the hour-long experiments. The same band fitting (masks, background and constraints) is performed on all the spectra from different series and days using Igor PRO (Wavemetrics). Unless stated differently, the results presented in the figures correspond to averages of spectra taken with the same tip under the same conditions (of $E_{\mathrm{b}}$ and $I_{\mathrm{t}}$ ) during scans up and down, and the errors correspond to the standard deviations. The spectra are acquired with integration times of 5 seconds in Ar and of 30 seconds in water with a $600 \mu \mathrm{W}$ excitation power at the exit of the objective. In the figures, they are presented normalized to the same time and power. Note that signal losses are expected when working in liquid due to beam aberrations and far-field focus distortion. ${ }^{\mathbf{1 0 , 1 3}}$

\section{Results and discussion}

The system under study in this work is a monolayer of thiophenol $(\mathrm{PhS}$, inset Fig. 1) adsorbed at a Au(111) single crystal. This sample was chosen because of the strong chemisorption of $\mathrm{PhS}$ at Au through the $\mathrm{S}$ atom, providing stability to the monolayer and a strong and reproducible TER signal suitable for performing hour-long experiments where many combinations of STM parameters can be

A) $E_{\text {bias }}=0.04 \mathrm{~V}$ (constant)

B) $I_{t}=1.3 \mathrm{nA}$ (constant)

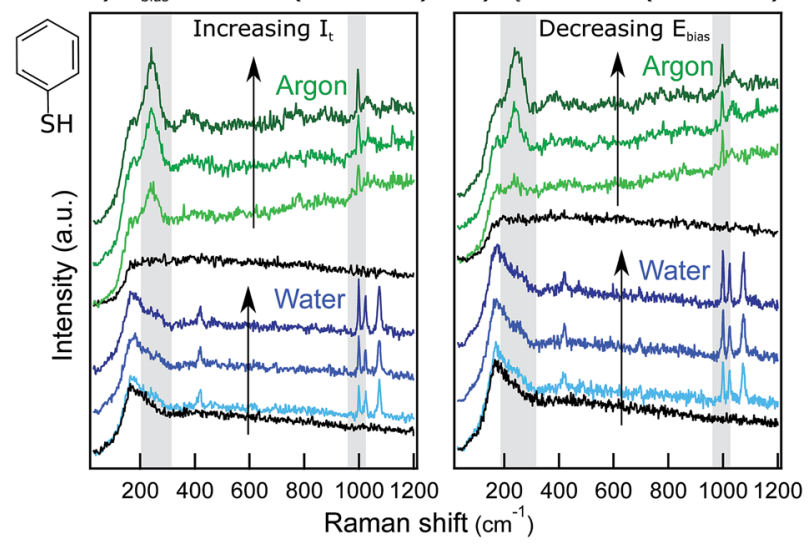

Fig. 1 Raw spectra recorded in $\operatorname{Ar}$ (green) and in water (blue) for $(A) I_{t}$ ramped at a constant $E_{\mathrm{b}}$ of $0.04 \mathrm{~V}$, (B) $E_{\mathrm{b}}$ ramped at a constant $I_{\mathrm{t}}$ of $1.3 \mathrm{nA}$. For each series, $20 \mathrm{~nm}$ retraction spectra (far-field) are included in black. Gray rectangles indicate marker bands. Spectra are $y$-offset for clarity. Inset: PhS chemical structure. 
explored with the same tip. Additionally, the TERS literature on PhS/Au(111) is extensive which facilitates band assignment and comparisons with previous results. ${ }^{9,13,27}$

Fig. 1 shows examples of raw TER spectra acquired during series with (A) varying $I_{\mathrm{t}}$ at a constant $E_{\mathrm{b}}=0.04 \mathrm{~V}$ and (B) varying $E_{\mathrm{b}}$ at a constant $I_{\mathrm{t}}=1.3 \mathrm{nA}$ in $\mathrm{Ar}$ (green) and in water (blue). The most prominent bands of PhS are visible with a high signal-to-noise ratio $(\mathrm{S} / \mathrm{N})$ in all the spectra and are in agreement with previous TERS reports for $\mathrm{PhS} / \mathrm{Au}(111) .^{9,13,27}$

All the spectra exhibit two prominent bands at $c a .245 \mathrm{~cm}^{-1}\left(\delta_{\mathrm{CS}}+\nu_{\mathrm{AuS}}\right)$ and at $997 \mathrm{~cm}^{-1}\left(\mathrm{r}-\mathrm{i}-\mathrm{d}+\nu_{\mathrm{CC}}\right)$. In Ar, the spectra also show two weak, broad bands at $c a$. $390 \mathrm{~cm}^{-1}\left(\gamma_{\mathrm{CH}}\right)$ and at $1024 \mathrm{~cm}^{-1}\left(\nu_{\mathrm{CC}}+\delta_{\mathrm{CH}}\right) .{ }^{28}$ The liquid spectra feature broad far-field bands at low wavenumbers (see the retracted spectra, black), partially masking the $245 \mathrm{~cm}^{-1} \mathrm{Au}-\mathrm{S}$ band that here is visible only as a shoulder. This farfield peak is present in all the liquid and electrochemical spectra acquired in our $\mathrm{lab}^{\mathbf{1 5}}$ and can be attributed to the aqueous work environment. In the water spectra, the peak at $1024 \mathrm{~cm}^{-1}\left(\nu_{\mathrm{CC}}+\delta_{\mathrm{CH}}\right)$ is more prominent than in $\mathrm{Ar}$, and an extra mode at $1074 \mathrm{~cm}^{-1}\left(\nu_{\mathrm{CC}}+\delta_{\mathrm{CH}}\right)$ shows up. The weak broad band at $390 \mathrm{~cm}^{-1}$ is not present in the liquid spectra, but an additional sharp peak appears at $c a$. $420 \mathrm{~cm}^{-1}\left(\nu_{\mathrm{CS}}+\nu_{\mathrm{AuS}}\right)$. In the literature, different TER spectral signatures for $\mathrm{PhS} /$ $\mathrm{Au}$ have been reported whose chemical origin has remained unclear. Additional experiments clarifying the physico-chemical interactions of $\mathrm{PhS} / \mathrm{Au}$ in $\mathrm{Ar}$ and in water are currently under way in our laboratory and will be published elsewhere. Here, we focus the discussion on the relative intensity changes of the $\mathrm{Au}-\mathrm{S}$ mode at $245 \mathrm{~cm}^{-1}$ and the ring-in-plane deformation mode at $997 \mathrm{~cm}^{-1}$ that are present both in the liquid and in the Ar spectra and are therefore ideal for singling out the influence of STM parameters in different environments. Additionally, given the technical specifications of our instrument, the mode at $245 \mathrm{~cm}^{-1}$ can be used as direct reporter on the effect of the sample potential on the adsorbate-substrate interaction.

Fig. 1A includes three example spectra at increasing $I_{\mathrm{t}}$ values (from bottom to top), both in $\mathrm{Ar}$ and in water. Increasing the tunneling current set-point results in an increase of the marker bands for both the Ar and water experiments. Similarly, ramping the bias voltage to lower values increases the intensity of the marker bands (Fig. 1B). These trends are in qualitative agreement with the expected decrease of the gap distance upon an $I_{\mathrm{t}}$ increase or $E_{\mathrm{b}}$ decrease. To gain quantitative insight into this relationship, Lorentzian band fittings have been performed for the different series and are discussed in the following sections.

\subsection{Influence of the tunneling current set-point, $I_{t}$, on the TER response}

Fig. 2 shows the integrated intensities of the $245 \mathrm{~cm}^{-1}$ (A) and $997 \mathrm{~cm}^{-1}$ (B) bands as a function of $\ln \left(I_{\mathrm{t}}\right)$ for constant $E_{\mathrm{b}}$ in $\operatorname{Ar}(0.02 \mathrm{~V}$, green circles; $0.04 \mathrm{~V}$, dark blue squares; $0.1 \mathrm{~V}$, purple triangles) and in water (0.04 V, light blue diamonds). A logarithmic $x$-axis scale has been chosen because of the gap-distance dependence on $I_{\mathrm{t}}$ and $E_{\mathrm{b}}$ according to eqn (2). Note that in a linear scale, $I_{\mathrm{TERS}}$ as a function of $I_{\mathrm{t}}$ shows a linear increase upon current increase (see ESI, $\uparrow$ Section 1). All the Ar results presented in this figure were acquired on the same day and with the same tip at different locations on a flat $\mathrm{Au}(111)$ terrace while the liquid results were taken on a different day with a different tip. 

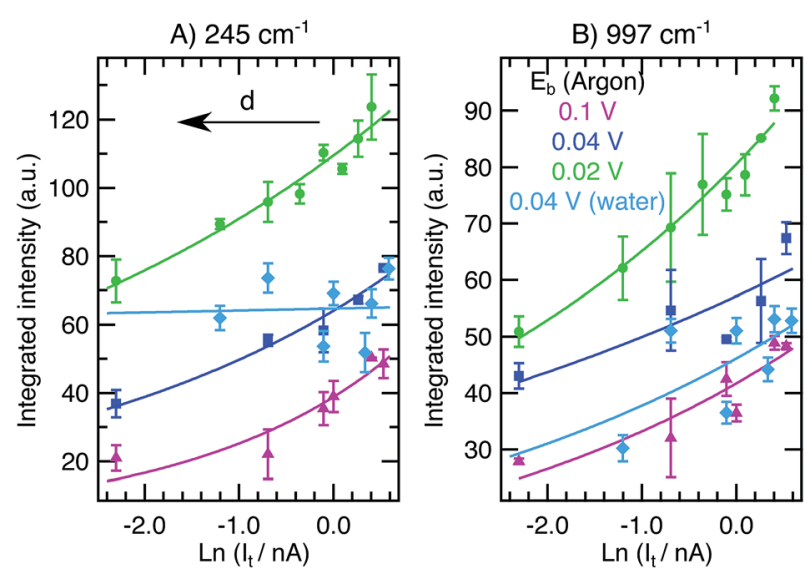

Fig. 2 (A) Integrated intensity of the $245 \mathrm{~cm}^{-1}$ mode ((B) $\left.997 \mathrm{~cm}^{-1}\right)$ as a function of $\ln (/ / \mathrm{t})$ at constant $E_{\mathrm{b}}$ values of $0.02 \mathrm{~V}$ (green circles), $0.04 \mathrm{~V}$ (dark blue squares), and $0.1 \mathrm{~V}$ (purple triangles) in $\mathrm{Ar}$ and $0.04 \mathrm{~V}$ in water (light blue diamonds). Averaged results from spectra taken in scans up and down are presented with the standard deviations as the errors. Fittings to eqn (3) are included (solid lines).

We find that $I_{\text {TERS }}$ increases upon current increase as expected from a gap distance reduction (eqn (1)-(3)). The value of $E_{\mathrm{b}}$ applied while varying the current has an influence on the absolute TERS intensity. For higher voltages $(0.1 \mathrm{~V}$, purple), the overall intensity detected is lower than for lower values $(0.02 \mathrm{~V}$, green $)$ in agreement with the larger tip-sample gap expected at high $E_{\mathrm{b}}$. While there are small differences in the growth rates between the two bands and between different constant values of $E_{\mathrm{b}}$ (see ESI, $\uparrow$ Section 1), the average increase in intensity in the scanned set-point range is $87 \%$ in $\mathrm{Ar}$ and $76 \%$ in water. Therefore in a TERS experiment, higher values of tunneling current are preferred if the maximum enhancement is sought both in Ar and liquid experiments. Tuning the current to large values (larger than $1 \mathrm{nA}$ ) decreases the gap distance and achieves very high intensities without inducing any obvious chemical change that might further alter the signal intensity or band position (see ESI, $\dagger$ Section 2 for discussion of the Raman shifts) while the tip-sample separation is still large enough to prevent tip crash or contamination.

\subsection{Influence of the bias voltage, $E_{\mathrm{b}}$, on the TER response}

Fig. 3 displays the integrated intensities of the $245 \mathrm{~cm}^{-1}$ (A) and $997 \mathrm{~cm}^{-1}$ (B) bands as a function of $\ln \left(E_{\mathrm{b}}\right)$ for constant values of the tunneling current set-point in $\mathrm{Ar}$ ( $0.3 \mathrm{nA}$, orange triangles; $0.8 \mathrm{nA}$, red circles; $1.3 \mathrm{nA}$, black squares) and water (1.3 nA, light blue diamonds).

Decreasing $E_{\mathrm{b}}$ reduces the tip-sample gap size and therefore results in an increase of $I_{\text {TERS }}$ (eqn (1)-(3)) as reported in Fig. 3. From the figure, it is apparent that the choice of $I_{\mathrm{t}}$ has a negligible effect on the TERS intensity over the investigated $E_{\mathrm{b}}$ range, in contrast to ramping $I_{\mathrm{t}}$ where $I_{\mathrm{TERS}}$ was higher for lower $E_{\mathrm{b}}$ values. The orange, red and black curves in Fig. 3B for the $997 \mathrm{~cm}^{-1}$ peak (taken at $0.3,0.8$ and $1.3 \mathrm{nA}$, respectively) overlap. For the $245 \mathrm{~cm}^{-1}$ peak, the overall intensity at $1.3 \mathrm{nA}$ is lower than the intensities at $0.3 \mathrm{nA}$ and $0.8 \mathrm{nA}$. On average 

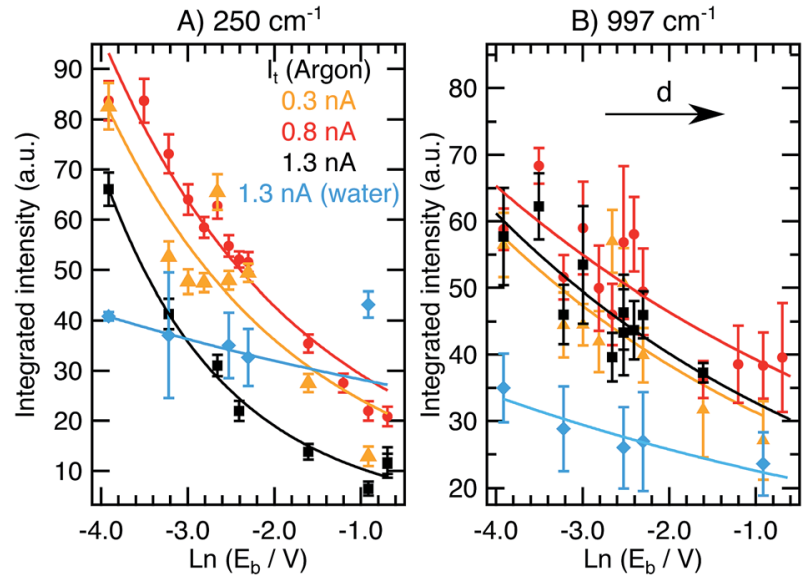

Fig. 3 (A) Integrated intensity of the $245 \mathrm{~cm}^{-1}$ mode ((B) $\left.997 \mathrm{~cm}^{-1}\right)$ as a function of $E_{\mathrm{b}}$ at constant $I_{\mathrm{t}}$ values of $0.3 \mathrm{nA}$ (orange triangles), $0.8 \mathrm{nA}$ (red circles), and $1.3 \mathrm{nA}$ (black squares) in $\mathrm{Ar}$ and $1.3 \mathrm{nA}$ in water (light blue diamonds). Averaged results from spectra taken in scans up and down are presented with the standard deviations as the errors. Fittings to eqn (3) are included (solid lines).

(considering both bands and the different values of constant $I_{\mathrm{t}}$ applied), the intensity increase achieved during the bias ramp is $263 \%$ in $\mathrm{Ar}$ and $47 \%$ in liquid, in contrast with the values of $87 \%$ in $\mathrm{Ar}$ and $76 \%$ in water obtained in the variable current series presented in previous section.

Given these numbers, we conclude that tuning $E_{\mathrm{b}}$ between 0.02 and $0.5 \mathrm{~V}$ has a much stronger effect on the gap distance and on the TER signal intensity than ramping $I_{\mathrm{t}}$ in the range between 0.3 and $1.3 \mathrm{nA}$. This effect is obvious when plotting the data in Fig. 3 as a function of $E_{\mathrm{b}}$ in a linear scale and comparing it to $I_{\text {TERS }} v s$. $I_{\mathrm{t}}$ plots (see ESI, $\uparrow$ Section 1 ) where the former exhibits an exponential behavior and the latter a linear trend. When searching for the highest sensitivity in a TERS experiment, higher values of $I_{\mathrm{t}}$ (higher than $1 \mathrm{nA}$ ) and lower values of $E_{\mathrm{b}}$ (under $0.1 \mathrm{~V}$ ) are preferred, with $E_{\mathrm{b}}$ being the more critical parameter compared to $I_{\mathrm{t}}$ regarding the TER signal intensity. Interestingly, we find that the described effect on $I_{\text {TERS }}$ is smaller in water than it is in Ar for the given parameter space. Further investigations exploring ranges with $I_{\mathrm{t}}>1.7 \mathrm{nA}$ and $E_{\mathrm{b}}<0.02 \mathrm{~V}$ in water could provide more insight into this observation. In general, this first systematic qualitative evaluation allows us to quantitatively correlate the effects of both $I_{\mathrm{t}}$ and $E_{\mathrm{b}}$ on $I_{\mathrm{TERS}}$.

\subsection{Distance dependence model: fitting results}

To evaluate if the dependence of the TER signal intensity on the tunneling current set-point and bias voltage follows a simple distance dependence in the case of $\mathrm{PhS} / \mathrm{Au}(111)$, we fitted the experimental data from Fig. 2 and 3 with eqn (3) (solid lines in the figures) in a free fitting procedure, obtaining $\rho, \beta$ and $R\left(z_{0}\right)$ for each of the series. The averaged fitting results are summarized in Table 1 for the current set-point and bias voltage ramps, for different bands and different values of $I_{\mathrm{t}}$ and $E_{\mathrm{b}}$ from two different experiments in Ar (with different tips) and one in water. All 
Table 1 Averaged fitfing parameters for the data points in Fig. 2 and 3. Dipole radius $\rho$; decay constant $\beta=\frac{4 \pi}{h} \sqrt{2 m \phi}$; and tunneling resistance at the landing position $R\left(z_{0}\right)$

\begin{tabular}{lccc}
\hline & $\rho / \mathrm{nm}$ & $\beta / \AA^{-1}$ & $R\left(z_{0}\right) / \mathrm{M} \Omega$ \\
\hline $\operatorname{Ar}$ (day 1) & $14 \pm 3$ & $0.27 \pm 0.07$ & $34.5 \pm 20$ \\
Ar (day 2) & $17 \pm 6$ & $0.36 \pm 0.12$ & $24.5 \pm 10$ \\
Ar (average) & $16 \pm 2$ & $0.32 \pm 0.05$ & $30 \pm 7$ \\
Water & $20 \pm 2$ & $0.33 \pm 0.04$ & $16 \pm 4$
\end{tabular}

the fittings were performed with the same initial guesses of the fitting parameters $\left(\rho=15 \mathrm{~nm}, \beta=0.3 \AA^{-1}\right.$ and $\left.R\left(z_{0}\right)=50 \mathrm{M} \Omega\right)$. The function accurately describes the experimental data in all cases. The obtained values for the tip radius (i.e. dipole radius $\rho$ ) from different days, on average $17 \pm 3 \mathrm{~nm}$, are in agreement with the average tip sizes produced in our lab as estimated from SEM images. ${ }^{13}$

From fitting, we obtain $\beta$ values of 0.32 and $0.33 \AA^{-1}$ in air and in water, respectively. The tunneling decay constant, $\beta$, has been measured experimentally for a number of similar molecules in previous works. Frisbie et $a .^{29}$ found an average $\beta=0.4 \AA^{-1}$ for alkanethiols of different lengths, with a tip-to-tip variation in the same range as we find for different days. In ref. $24, \beta=0.27 \AA^{-1}$ was reported for an azobenzene monolayer on $\mathrm{Au}(111)$. Electrochemical methods applied to similar aromatic molecules give values of $\beta$ between 0.35 and $0.57 \AA^{-1} .^{30,31}$ Our $\beta$ values lie well within the reported variability. Small discrepancies may be due to differences in the chemical structure, orientation or packing of the monolayer that directly affect the electron transport in the junction. ${ }^{24,32,33}$ Interestingly, the obtained decay constant values for experiments in $\mathrm{Ar}$ and in water are very similar, despite the smaller values of work function that are expected in a liquid. ${ }^{34-36}$

The values for the junction resistance at the landing position obtained from fitting are 30 and $16 \mathrm{M} \Omega$ for air and liquid, respectively. Contact resistance $R\left(-\mathrm{z}_{0}\right) \ddagger$ literature values between 0.1 and $50 \mathrm{M} \Omega$ have been found for similar molecules, among which a value of $\approx 0.1 \mathrm{M} \Omega$ is found for the $\mathrm{PhS} / \mathrm{Au}(111)$ system. ${ }^{29,37}$ Higher values are expected for $R\left(z_{0}\right)$ since here the molecular length and gap distance are also considered. Toccafondi et al. ${ }^{24}$ recently reported $R\left(z_{0}\right)$ to be $694 \mathrm{M} \Omega$ for azobenzene/ $\mathrm{Au}(111)$ in a $E_{\mathrm{b}}$-dependence TERS study. Compared to the literature values, the experimental values of 30 and $16 \mathrm{M} \Omega$ that we obtain in $\mathrm{Ar}$ and in water, respectively, lie well within the expected range, considering that $\mathrm{PhS}$ is shorter than azobenzene. Furthermore, a lower resistance in water is in agreement with the better electric conductivity of MilliQ (resistivity of $c a .18 \mathrm{M} \Omega$ ) compared to Ar.

In summary, as the fittings quantitatively describe our experimental data points, and the values of the fitting parameters obtained are in agreement with literature values, for both Ar and water and for current set-point and voltage ramps, the TER signal intensity follows the expected distance dependence relationship in eqn (3) for $I_{\mathrm{t}}$ and $E_{\mathrm{b}}$ ramps, both in Ar and in water. Additional

\$ While $R\left(-z_{0}\right)$ accounts for the resistance of the junction in contact between the tip and sample, the parameter in eqn (3), $R\left(z_{0}\right)$, is the junction resistance at the landing position. 

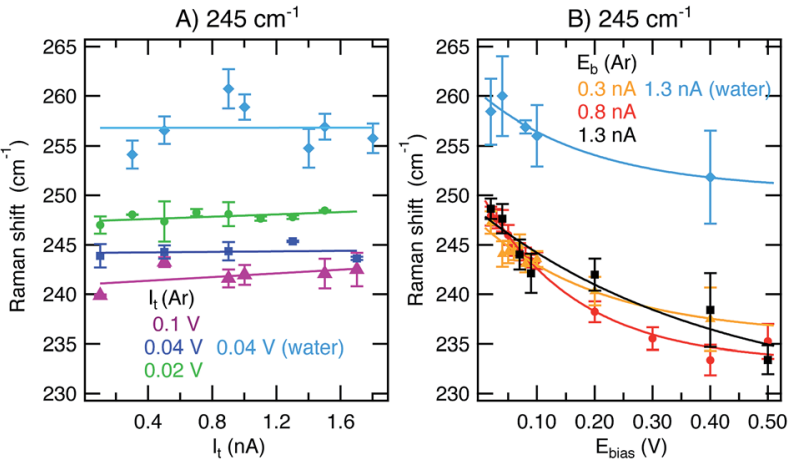

Fig. 4 Raman shift of the $245 \mathrm{~cm}^{-1}$ mode as a function of $I_{\mathrm{t}}\left((\mathrm{A})\right.$, at constant $E_{\mathrm{b}}$ values of $0.02 \mathrm{~V}$, green circles; $0.04 \mathrm{~V}$, dark blue squares; $0.1 \mathrm{~V}$ in $\mathrm{Ar}$, purple triangles; and $0.04 \mathrm{~V}$ in water, light blue diamonds) or $E_{\mathrm{b}}\left((\mathrm{B})\right.$, at constant $I_{\mathrm{t}}$ values of $0.3 \mathrm{nA}$, orange triangles; $0.8 \mathrm{nA}$, red circles; $1.3 \mathrm{nA}$ in Ar, black squares; and $1.3 \mathrm{nA}$ in water, light blue diamonds). Averaged results from spectra taken in scans up and down are presented with the standard deviation as the error. Solid lines are included as a guide to the eye.

geometric effects such as molecular bending that have been reported for longer molecules ${ }^{24}$ do not need to be taken into account in the case of $\mathrm{PhS} / \mathrm{Au}(111)$.

\subsection{Raman shift of the Au-S mode}

In addition to changes in $I_{\text {TERS }}$, we also observe a shift of the $245 \mathrm{~cm}^{-1}$ band as a function of $E_{\mathrm{b}}$. In the series of measurements at constant $E_{\mathrm{b}}$ and variable $I_{\mathrm{t}}$ represented in Fig. 4A, the $245 \mathrm{~cm}^{-1}$ mode appears at a constant position throughout the scanned set-point range. However, the peak maximum is shifted to lower wavenumbers upon $E_{\mathrm{b}}$ increase. This effect is obvious in the series of measurements at constant $I_{\mathrm{t}}$ and variable bias presented in Fig. 4B. The position of the band at $245 \mathrm{~cm}^{-1}$ experiences a large red-shift upon bias increase independently of the environment or the value of constant set-point applied. The total shift within the potential window scanned is $c a .14 \mathrm{~cm}^{-1}$. Note that no notable Raman shift is detected for the $997 \mathrm{~cm}^{-1}$ band (see ESI, $\dagger$ Section 2).

The fact that only the $245 \mathrm{~cm}^{-1}$ band shifts and only when $E_{\mathrm{b}}$ is ramped can be explained by the changing interaction strength of the S-Au bond when ramping the substrate potential ( $E_{\mathrm{b}}$ here due to the technical specifications of our instrument). Upon decreasing $E_{\mathrm{b}}$, the $\mathrm{Au}(111)$ electrode is more positively charged, in this way enabling stronger $\pi(\mathrm{S})-\mathrm{d}^{*}(\mathrm{Au})$ overlap and increased binding strength. A similar effect has been found in an electrochemical TERS study for the $\mathrm{N}-\mathrm{Au}$ interaction of adenine/Au(111). ${ }^{15}$ While the paper at hand focuses on the influence of the STM parameters on the TER signature, it would be interesting to quantify the binding strength between $\mathrm{PhS}$ and $\mathrm{Au}$ as a function of the substrate potential in future experiments and theoretical simulations.

\section{Conclusions}

We have systematically studied the behaviour of STM-TER spectra as a function of the bias voltage and tunneling current set-point in experiments in $\mathrm{Ar}$ and in water. 
In both cases, for the PhS/Au(111) system, $I_{\text {TERS }}$ follows a dependence on the STM parameters expected from a simple gap-distance reduction upon set-point increase or bias decrease. In water, the intensity changes are more moderate than in the Argon experiments. Regarding the absolute intensity increase, tuning the bias voltage in the 0.5 to $0.02 \mathrm{~V}$ range results in an exponential band intensity increase while tuning the current set-point in $\mathrm{Ar}$ in the range from 0.1 to $1.7 \mathrm{nA}$ shows a linear dependence. In practice, both parameters can be tuned to achieve optimal enhancement factors in STM-TERS experiments with the best results obtained with $I_{\mathrm{t}}>1 \mathrm{nA}$ and $E_{\mathrm{b}}<0.1 \mathrm{~V}$ for the given $\mathrm{PhS} / \mathrm{Au}(111)$ system under study. If the bias voltage is changed by tuning the potential of the sample with respect to the tip - as is often the case with commercial STMs - the interaction strength of the PhS-Au bond is altered when changing $E_{\mathrm{b}}$, which manifests itself as a change in the Raman shift of the Au-S stretch vibration while the other band positions in the spectrum remain unchanged. We conclude by pointing out the possibilities that a smart choice of STM parameters presents for ultrasensitive TERS studies of solid/liquid interfaces and/or single molecules.

\section{Acknowledgements}

KFD is grateful for generous financial support of this work through the Emmy Noether program of the Deutsche Forschungsgemeinschaft (grant no. DO1691/11) and through the FP7 Marie Curie Actions ITN-FINON (grant no. 607842). The authors thank Leonie Driessen, Matthias Fenner, Marc-Jan van Zadel and the MPIP electronic workshop for discussions and smart solutions to TERS technical challenges. Open Access funding provided by the Max Planck Society.

\section{References}

1 N. Jiang, D. Kurouski, E. A. Pozzi, N. Chiang, M. C. Hersam and R. P. Van Duyne, Chem. Phys. Lett., 2016, 659, 16-24.

2 A. Hartschuh, Angew. Chem., Int. Ed., 2008, 47, 8178-8191.

3 K. F. Domke, D. Zhang and B. Pettinger, J. Am. Chem. Soc., 2006, 128, 1472114727.

4 W. Zhang, B. S. Yeo, T. Schmid and R. Zenobi, J. Phys. Chem. C, 2007, 111, 1733-1738.

5 R. Zhang, Y. Zhang, Z. C. Dong, S. Jiang, C. Zhang, L. G. Chen, L. Zhang, Y. Liao, J. Aizpurua, Y. Luo, J. L. Yang and J. G. Hou, Nature, 2013, 498, 82-86.

6 N. Chiang, X. Chen, G. Goubert, D. V. Chulhai, X. Chen, E. A. Pozzi, N. Jiang, M. C. Hersam, T. Seideman, L. Jensen and R. P. Van Duyne, Nano Lett., 2016, 16, 7774-7778.

7 A. B. Zrimsek, N. Chiang, M. Mattei, S. Zaleski, M. O. McAnally, C. T. Chapman, A.-I. Henry, G. C. Schatz and R. P. Van Duyne, Chem. Rev., 2017, 117(11), 75837613.

8 J.-H. Zhong, X. Jin, L. Meng, X. Wang, H.-S. Su, Z.-L. Yang, C. T. Williams and B. Ren, Nat. Nanotechnol., 2016, 12, 132-136.

9 T. Schmid, B.-S. Yeo, G. Leong, J. Stadler and R. Zenobi, J. Raman Spectrosc., 2009, 40, 1392-1399.

10 B. Gjonaj, P. Johnson, M. Bonn and K. F. Domke, Appl. Opt., 2012, 51, 80348040. 
11 Z.-C. Zeng, S.-C. Huang, D.-Y. Wu, L.-Y. Meng, M.-H. Li, T.-X. Huang, J.-H. Zhong, X. Wang, Z.-L. Yang and B. Ren, J. Am. Chem. Soc., 2015, 137, 11928-11931.

12 D. Kurouski, M. Mattei and R. P. Van Duyne, Nano Lett., 2015, 15, 7956-7962.

13 N. Martín Sabanés, L. Driessen and K. F. Domke, Anal. Chem., 2016, 88, 71087114.

14 M. Mattei, G. Kang, G. Goubert, D. V. Chulhai, G. C. Schatz, L. Jensen and R. P. Van Duyne, Nano Lett., 2016, 17, 590-596.

15 N. Martín Sabanés, T. Ohto, D. Andrienko, Y. Nagata and K. F. Domke, Angew. Chem. Int. Ed., 2017, 56, 9796-9801.

16 B. Pettinger, K. F. Domke, D. Zhang, G. Picardi and R. Schuster, Surf. Sci., 2009, 603, 1335-1341.

17 I. Notingher and A. Elfick, J. Phys. Chem. B, 2005, 109, 15699-15706.

18 B. Pettinger, K. F. Domke, D. Zhang, R. Schuster and G. Ertl, Phys. Rev. B: Condens. Matter Mater. Phys., 2007, 76, 113409.

19 L. G. Cançado, A. Jorio, A. Ismach, E. Joselevich, A. Hartschuh and L. Novotny, Phys. Rev. Lett., 2009, 103, 1861011-1861014.

20 R. V. Maximiano, R. Beams, L. Novotny, A. Jorio and L. G. Cançado, Phys. Rev. B: Condens. Matter Mater. Phys., 2012, 85, 1-8.

21 J. G. Simmons, J. Appl. Phys., 1963, 34, 1793-1803.

22 E. Meyer, H. J. Hug and R. Bennewitz, Scanning Probe Microscopy: the lab on a tip, Springer-Verlag, Berlin Heildelberg GmbH, 1st edn, 2004.

23 R. L. McCreery, Chem. Mater., 2004, 16, 4477-4496.

24 C. Toccafondi, G. Picardi and R. Ossikovski,J. Phys. Chem. C, 2016, 120, 18209-18219.

25 Y. Fujita, N. N. Horimoto, S. Kajimoto and H. Fukumura, Chem. Phys. Lett., 2013, 582, 110-114.

26 J. Clavilier, R. Faure, G. Guinet and R. Durand, J. Electroanal. Chem. Interfacial Electrochem., 1979, 107, 205-209.

27 C. Blum, L. Opilik, J. M. Atkin, K. Braun, S. B. Kämmer, V. Kravtsov, N. Kumar, S. Lemeshko, J. F. Li, K. Luszcz, T. Maleki, A. J. Meixner, S. Minne, M. B. Raschke, B. Ren, J. Rogalski, D. Roy, B. Stephanidis, X. Wang, D. Zhang, J. H. Zhong and R. Zenobi, J. Raman Spectrosc., 2014, 45, 22-31.

28 C. G. T. Feugmo and V. Liégeois, ChemPhysChem, 2013, 14, 1633-1645.

29 D. J. Wold, R. Haag, M. A. Rampi and C. D. Frisbie, J. Phys. Chem. B, 2002, 106, 2813-2816.

30 S. B. Sachs, S. P. Dudek, R. P. Hsung, L. R. Sita, J. F. Smalley, M. D. Newton, S. W. Feldberg and C. E. D. Chidsey, J. Am. Chem. Soc., 1997, 119, 10563-10564.

31 S. Creager, C. J. Yu, C. Bamdad, S. O. Connor, T. Maclean, Y. Chong, G. T. Olsen, J. Luo, M. Gozin, J. F. Kayyem and E. Lam, J. Am. Chem. Soc., 1999, 121, 1059-1064.

32 H. Yamamoto and D. H. Waldeck, J. Phys. Chem. B, 2002, 106, 7469-7473.

33 L. Venkataraman, J. E. Klare, C. Nuckolls, M. S. Hybertsen and M. L. Steigerwald, Nature, 2006, 442, 904-907.

34 J. Pan, T. W. Jing and S. M. Lindsay, J. Phys. Chem., 1994, 98, 4205-4208.

35 J. Halbritter, G. Repphun, S. Vinzelberg, G. Staikov and W. J. Lorenz, Electrochim. Acta, 1995, 40, 1385-1394.

36 M. Hugelmann and W. Schindler, Surf. Sci., 2003, 541, L643-L648.

37 H. H. B. Akkerman, B. de Boer and B. D. Boer, J. Phys.: Condens. Matter, 2008, 20, 13001. 